Swarthmore College

Works

$10-7-2018$

\title{
A Computational Study Of The Role Of Spatial Receptive Field Structure In Processing Natural And Non-Natural Scenes
}

Victor J. Barranca

Swarthmore College, vbarran1@swarthmore.edu

G. Zhu

Follow this and additional works at: https://works.swarthmore.edu/fac-math-stat

Part of the Mathematics Commons

Let us know how access to these works benefits you

\section{Recommended Citation}

Victor J. Barranca and G. Zhu. (2018). "A Computational Study Of The Role Of Spatial Receptive Field Structure In Processing Natural And Non-Natural Scenes". Journal Of Theoretical Biology. Volume 454, 268-277. DOI: 10.1016/j.jtbi.2018.06.011

https://works.swarthmore.edu/fac-math-stat/236

This work is brought to you for free by Swarthmore College Libraries' Works. It has been accepted for inclusion in Mathematics \& Statistics Faculty Works by an authorized administrator of Works. For more information, please contact myworks@swarthmore.edu. 


\section{Accepted Manuscript}

A Computational Study of the Role of Spatial Receptive Field Structure in Processing Natural and Non-Natural Scenes

Victor J. Barranca, George Zhu

PII:

S0022-5193(18)30305-9

DOI:

10.1016/j.jtbi.2018.06.011

Reference:

YJTBI 9506

To appear in:

Journal of Theoretical Biology

Received date: $\quad 10$ April 2018

Revised date: $\quad 30$ May 2018

Accepted date: $\quad 12$ June 2018

Please cite this article as: Victor J. Barranca, George Zhu, A Computational Study of the Role of Spatial Receptive Field Structure in Processing Natural and Non-Natural Scenes, Journal of Theoretical Biology (2018), doi: 10.1016/j.jtbi.2018.06.011

This is a PDF file of an unedited manuscript that has been accepted for publication. As a service to our customers we are providing this early version of the manuscript. The manuscript will undergo copyediting, typesetting, and review of the resulting proof before it is published in its final form. Please note that during the production process errors may be discovered which could affect the content, and all legal disclaimers that apply to the journal pertain. 


\section{Highlights}

- Localized receptive fields and nonlinear dynamics give rise to illusory effects

- Center-surround receptive fields facilitate accurate encoding of natural scenes

- Model networks with several classes of receptive fields are proposed and compared

- A compressive sensing framework is developed for analyzing stimulus encoding

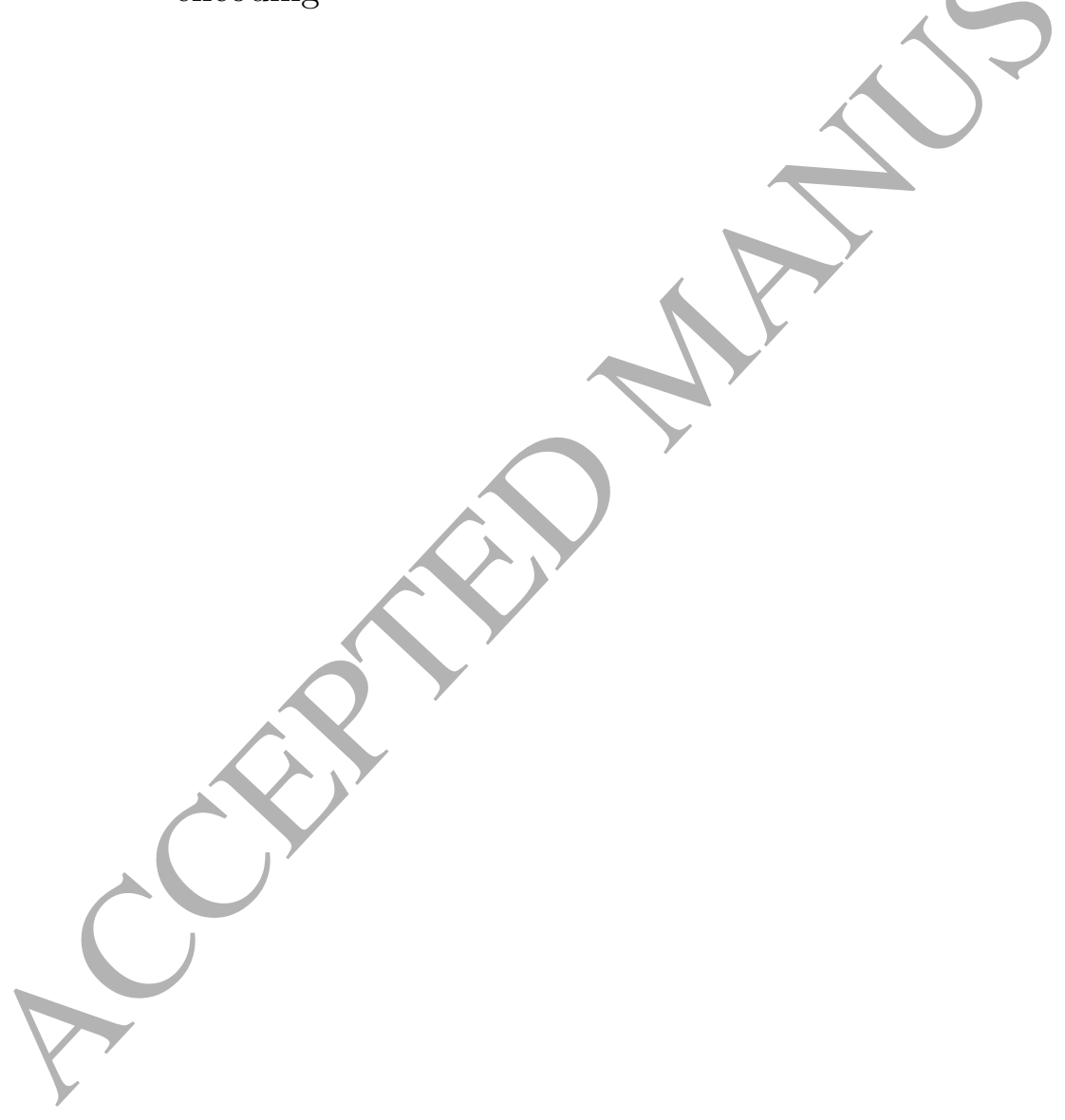




\title{
A Computational Study of the Role of Spatial Receptive Field Structure in Processing Natural and Non-Natural Scenes
}

\author{
Victor J. Barranca ${ }^{\mathrm{a}, *}$, George Zhu ${ }^{\mathrm{a}}$ \\ ${ }^{a}$ Swarthmore College, 500 College Avenue, Swarthmore, PA, 19081, USA
}

\section{Abstract}

The center-surround receptive field structure, ubiquitous in the visual system, is hypothesized to be evolutionarily advantageous in image processing tasks. We address the potential functional benefits and shortcomings of spatial localization and center-surround antagonism in the context of an integrate-andfire neuronal network model with image-based forcing. Utilizing the sparsity of natural scenes, we derive a compréssive-sensing framework for input image reconstruction utilizing evoked neuronal firing rates. We investigate how the accuracy of input encoding depends on the receptive field architecture, and demonstrate that spatial localization in visual stimulus sampling facilitates marked improvements in natural scene processing beyond uniformly-random excitatory connectivity. However, for specific classes of images, we show that spatial localization inherent in physiological receptive fields combined with information loss through nonlinear neuronal network dynamics may underlie common optical illusions, giving a novel explanation for their manifestation. In the context of signal processing, we expect this work may suggest new sampling protocols useful for extending conventional compressive sensing theory.

\section{Keywords:}

sensory processing; neuronal networks; nonlinear dynamics; optical illusions; compressive sensing

\footnotetext{
*vbarran1@swarthmore.edu
} 


\section{Introduction}

Numerous lines of evidence suggest that mammals have evolved with the goal of optimally encoding natural stimuli through the dynamics of neuronal networks [1-5]. Across diverse sensory spaces, specific stimulus characteristics are known to determine the firing activity of neurons, giving rise to rich receptive field structures $[6,7]$. These receptive fields manifest in different forms in the visual, somatosensory, auditory, and olfactory systems, though stimuli sharing key qualitatively similar characteristics tend to eyoke similar responses from a given sensory neuron [8-12].

In the early visual system in particular, the receptive field/structure is believed to play a crucial role in efficiently encoding image characteristics, such as contrast and spatial frequency composition [13-17]. Two key features observed in vivo in visual receptive fields are spatial localization and centersurround antagonism. In the context of the retina and lateral geniculate nucleus, a receptive field is known to typically take the form of two concentric circles, with stimulation in the inner circle and stimulation in the surrounding annulus having opposite effect. In the on-region, this stimulation increases the firing rate of a neuron, with stimulation in the off-region inhibiting activity, yielding an on-off or center-surround structure [6,7]. Thus, in this case, the response of a neuron to a particular stimulus depends on spatially local information about a small volume of visual space. Since receptive field size is generally heterogeneous, different receptive fields process information regarding disparate spatial frequency components of an image, with larger receptive fields typieally capturing lower frequency data [18-20]. Ganglion cells near the fovea, for example, generally have the smallest receptive fields with field sizes increasing in the periphery of the visual field [21, 22]. In this work, we take the perspective that only by capturing the full mosaic of dominant frequency information are visual stimuli well encoded [23, 24].

While the receptive field structure appears optimized for the processing of natural scenes, the receptive field architecture may also result in unforeseen difficulties in processing particular classes of images [25]. An illustrative example of this is the Hermann grid illusion displayed in Fig. 1A, comprised of intersecting horizontal and vertical white lines on a black grid [26]. At the squares of intersection between the white lines in the periphery, the image typically appears grey while it is in reality white. The classical explanation for this illusion is based on center-surround receptive fields [27, 28]. It is believed that the grey smudges are caused by ganglion cells sampling the center 


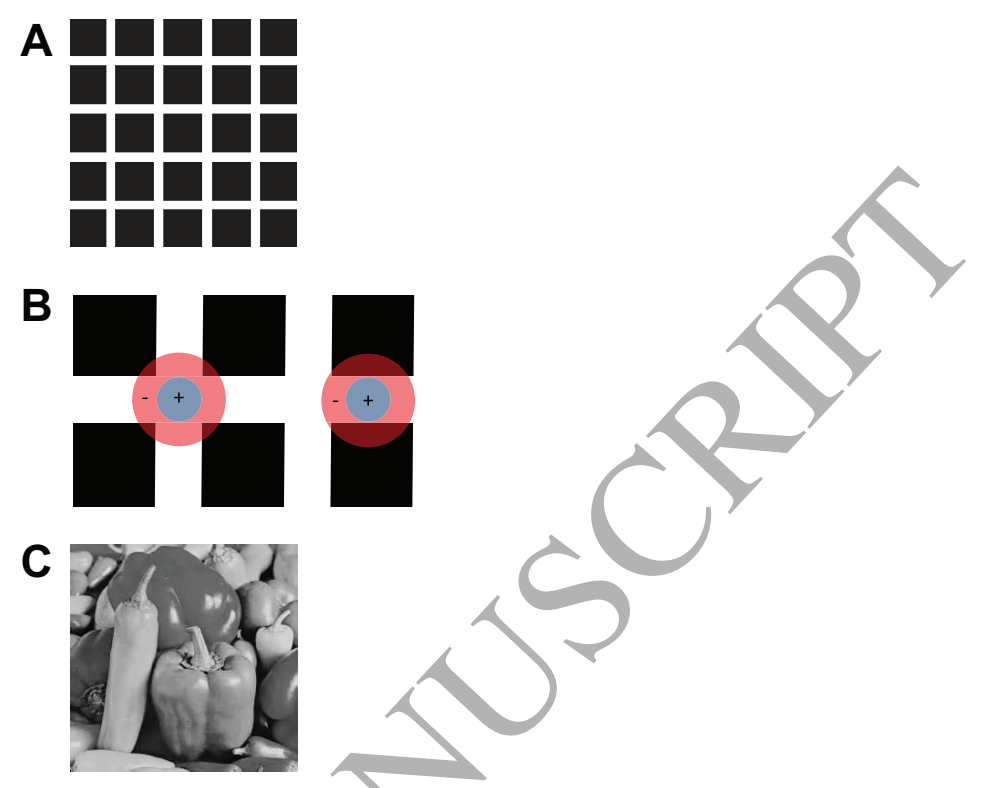

Figure 1: Stimulus types. (A) Hermann grid illusion. (B) Schematic explanation of the grid illusion using center-surround receptive field architecture. The left receptive field evokes a smaller response than the right receptive field. (C) Example natural scene.

of the intersections, thereby receiving more white-space input in the surround area relative to ganglion cells sampling other parts of the white lines. This extra white input, in an inhibitory surround region, would therefore decrease the firing activity of the ganglion cell and account for the mistaken inference that the intersections are grey. A schematic of this explanation is depicted in Fig. 1B.

We investigate the potential functional benefits and shortcomings of the spatial localization and center-surround paradigms embodying the receptive field structure in the visual system in the context of an integrate-and-fire neuronal network model. We view our network as a model of the early visual system, with input images sampled by the receptive fields of downstream neurons driving firing activity. Based on the sparsity of natural scenes, we utilize a compressive-sensing based theoretical framework for reconstructing stimuli from evoked neuronal firing rate dynamics [29]. The quality of the model input image reconstructions are used to gauge how well various classes of stimuli are encoded by the network dynamics. This framework thus gives a direct method of testing hypotheses regarding the manifestations of optical 
illusions that may currently otherwise be unverified in vivo.

We study how the accuracy of image encoding depends on the model receptive field structure and demonstrate that spatial localization does indeed facilitate marked improvements in natural stimulus encoding at the price of diminished encoding of non-natural scenes, such as the Hermann grid illusion. By optimizing over the parameter space of several receptive field models, we determine that an intermediate receptive field size yields optimal encoding of natural scenes, whereas the Hermann grid is best encoded by especially small receptive fields. Our work also indicates that spatial sampling localization, as opposed to the center-surround antagonism, could be a primary cause for the Hermann grid illusion. This investigation further underlines information loss through nonlinear network dynamics as an additional factor contributing to optical illusions. We expect that these connections between input characteristics, network topology, and neuronal dynamics will give new insights into the structure-function relationship of the visual system.

The organization of the paper is as follows. In Section 2.1, we introduce our mechanistic network model of the early visual system and then outline our input reconstruction framework using compressive sensing of the model network linear input-output relationship in Section 2.2. Next, in Section 2.3, we formulate several variant receptive field models, each incorporating various levels of biological realism, reflecting (i) uniformly random sampling as classical in CS theory, (ii) spatially localized sampling, and (iii) centersurround sampling, respectively. We systematically examine how each of these receptive field structures influences the reconstruction of both natural scenes and artificial illusory images in Section 3. Finally, in Section 4, we consider the implications of this work and possible directions for future investigation.

\section{Materials and Methods}

\subsection{Network Model}

To study how image information is encoded by sensory network dynamics given various receptive field structures, we construct an idealized two-layer model of the early visual system. An input image (replicating photoreceptor output) is sampled by a downstream neuronal network (ganglion cells) through a sampling matrix modeling the receptive fields of the downstream neurons. Since photoreceptors are known to exhibit graded potentials in response to incoming light [30], their output is fixed as a constant current 
vector with values corresponding to the pixels of a vectorized image. The input image evokes activity in the downstream layer of firing neurons, which we reflect using a current-based integrate-and-fire (I\&F) model [31-34].

In our network model, the subthreshold voltage dynamics of the $i^{\text {th }}$ downstream neuron is governed by dynamical system

$$
\tau \frac{d v_{i}}{d t}=-\left(v_{i}-V_{R}\right)+\frac{1}{n} \sum_{j=1}^{n} F_{i j} p_{j}+\frac{S}{N_{R}} \sum_{\substack{k=1 \\ k \neq i}}^{m} R_{i k} \sum_{l} \delta\left(t-\tau_{k l}\right),
$$

evolving from the reset potential $V_{R}$ until reaching the threshold potential $V_{T}$. At this time, the neuron is considered to have fired, and we reset $v_{i}$ to $V_{R}$, injecting the currents $\left(S / N_{R}\right) \delta\left(t-\tau_{i l}\right)$ into all the other downstream neurons post-connected to it, with $\delta(\cdot)$ the Dirac delta function and $\tau_{i l}$ the $l^{\text {th }}$ firing time of the $i^{\text {th }}$ downstream neuron.

Recurrent connections among the downstream neurons are described by an $m \times m$ matrix $R$, where $S$ is the recurrent connection strength and $N_{R}$ is the total number of recurrent connections. The input image is modeled by an $n$-vector $p$, which is sampled by the sparse feed-forward $m \times n$ connection matrix $F$. We assume the time scale for the dynamics is $\tau=20 \mathrm{~ms}$, as typical in vivo [35-37], and the dimensionless potential values $V_{R}=0, V_{T}=1$, and $S=1$. Note that the pixel values in $p$ are chosen to be $\mathcal{O}(1)$ quantities, with higher values denoting more white pixels. Normalizing the feed-forward input by $n$ assures that even as the number of pixels in the input image increase, the net input into a given downstream neuron is $\mathcal{O}(1)$. Similarly, normalizing the recurrent input by $N_{R}$ allows the expected recurrent input into a downstream neuron to remain constant even if the recurrent connection density is varied. We simulate this model using an event-driven algorithm in which we analytically and iteratively solve for neuronal voltages and spike times [38].

In the retina in particular, we note that while it was previously thought that retinal ganglion cells are generally uncoupled, relatively recent experimental evidence suggests there may be structural connections among specific types of ganglion cells, often in the form of gap junctions [39-44]. In the context of our mechanistic model network, which may similarly reflect alternative early sensory systems, we consider pulse-coupled interactions among the downstream neurons to maintain some generality and analytical tangibility. We select the elements of recurrent connectivity matrix $R$ to be independent identically distributed random variables, with each described by a Bernoulli 
distribution. As long as the recurrent coupling is not too strong, previous work demonstrates that the nonlinear network dynamics generally render a robust encoding of detailed network inputs. In fact, removing the recurrent connectivity completely has minimal impact on the reconstruction framework. Aside from possible interactions among recurrent neurons, we may alternatively view the pulse-coupling as a potential noise source due, for example, to fluctuations in photon absorption [45]. An alternative modeling assumption, adding independent and identically distributed Gaussian noise to each stimulus component, results in reconstruction error growing approximately linearly with the variance of the noise [29].

Reflecting the relatively large number of photoreceptors compared to the number of downstream ganglion cells in the retina $[46,47]$, we generally assume the model network is composed of $n=10^{4}$ upstream neurons and $m=10^{3}$ downstream neurons. In this sense, we may view the dynamics of the downstream neurons as a compressive encoding of the input image. We will elaborate on several receptive field models determined by feed-forward connectivity matrix $F$ in Section 2.3 and later examine how they influence the encoding of various classes of images in Section 3.

\subsection{Reconstruction of Network Inputs}

To reconstruct input images from network dynamics, we first derive a linear input-output mapping between the input images and evoked neuronal firing rates, and then we choose the optimal reconstruction through compressing sensing on the resultant underdetermined linear system.

Utilizing a nonequilibrium statistical mechanics approach, we coarse-grain the nonlinear network dynamics in the relatively high firing-rate regime and obtain the linear input-output mapping

$$
\sum_{j=1}^{n} F_{i j} p_{j}=\left(\tau \mu_{i}+\frac{1}{2}\right)\left(V_{T}-V_{R}\right)-\frac{S}{N_{R}} \sum_{k=1, k \neq i}^{m} R_{i k} \mu_{k}
$$

valid when the neuronal firing rates, $\mu_{j}$, are relatively high, such that $\mu_{j} \gg 1$ for all $j$, and the voltage jump induced by each spike is small such that $S / N_{R} \ll 1[29,48-50]$. Assuming the neuronal firing rates are known from model simulation (or experiment), then Eq. 2 can be viewed as an underdetermined linear system with unknown input signal $p$ sampled by the feedforward connectivity matrix $F$. 
From conventional signal processing theory, if image $p$ is uniformly sampled, then we can expect the number of samples (rows in $F$ ) necessary for a successful reconstruction of $p$ to be determined by its bandwidth [51]. However, if $p$ is sparse in a particular domain, then compressive sensing (CS) theory asserts that instead the number of necessary samples is determined by the sparsity of $p[52,53]$. The CS framework facilitates a high fidelity reconstruction of $p$ from Eq. 2 even though the coarse-grained linear system is highly underdetermined in the physiological case when the number of receptors is significantly larger than the number of downstream ganglion cells $(n \gg m)$.

In accordance with compressive sensing, we seek among the infinite number of solutions the particular solution with the smallest number of non-zero components in the frequency domain, which is thereby most compressible and efficiently represented by the evoked network activity. This translates to determining the solution to an $L_{1}$ optimization problem, which can efficiently be solved in polynomial time through, for example, the orthogonal matching pursuit $[29,54]$. Assuming image $p$ has a sparse representation in an appropriate domain, such that it is sparse under transform $T$ and thus $T(p)=\hat{p}$ with $\hat{p}$ sparse, the CS theory prescribes that the optimal reconstruction is achieved by satisfying Eq. 2 while minimizing $|\hat{p}|_{L_{1}}=\sum_{i=1}^{n}\left|\hat{p}_{i}\right|$. Considering natural images are known to be sparse in the frequency domain [1], this approach is highly successful for a large class of images. In our particular work, we utilize the two-dimensional discrete cosine transform as the sparsifying transformation, but similar results are achieved for alternative sparsifying transforms, such as the two-dimensional discrete Fourier transform and twodimensional discrete wavelet transform [55-58].

The quality of the reconstruction of $p$ thus can be considered an indication of how well the network dynamics encode a particular input. CS theory shows that sampling matrices exhibiting little correlation among their columns and well-preserving signal magnitudes, such as matrices with a sufficient degree of randomness in their structure [53, 59], are typically viable candidates. The success of the network input reconstruction is as a result dependent on the structure of the sampling matrix, the feed-forward connectivity matrix $F$ in this case, which we will investigate in the subsequent sections. We emphasize that the specific mapping we derive given by Eq. 2 does intrinsically yield error in our CS reconstructions. However, for CS theory to apply, the system 
considered must at least be well approximated by a static linear input-output mapping. Alternative mappings may yield distinct CS reconstructions, but in this work we take the perspective that, regardless of the linear map utilized, some encoding error is induced by the nonlinear neuronal dynamics. We argue this error may be fundamental in fully explaining illusory effects observed in certain classes of non-natural images.

\subsection{Classes of Receptive Field Models}

We will differentiate among three different receptive field types, comparing their utility in encoding various classes of images, including natural scenes and optical illusions. We consider a natural scene to be an image that a mammal would typically encounter as a visual stimulus as it traverses the physical environment in which it lives. Natural stimuli, such as the peppers image depicted in Fig. 1C demonstrate a high degree of correlation between neighboring pixels with generally smooth transitions among edges [60], which contrasts from the Hermann grid illusion image in Fig. 1A, which demonstrates rather abrupt transitions between black and white pixels.

Standard compressive sensing theory suggests that the feed-forward connectivity should be uniformly random to produce an optimal input image reconstruction, with each entry of feed-forward connectivity matrix $F$ an independent identically distributed Bernoulli random variable with connection probability $P$ and connection strength $f$. This simple receptive field type would be completely devoid of spatial structure, and less realistic relative to the early visual system. Note that the receptive field of the $i^{\text {th }}$ downstream neuron is modeled by the weighted pixel measurements composing the $i^{\text {th }}$ row of $F$.

A more realistic alternative receptive field model is localized random sampling. In this case, each receptive field is assumed to be randomly centered about a spatial location on the input image, with the probability of measuring a given nearby pixel decreasing with distance from the receptive field center. This sampling structure has sufficient randomness such that the rows are $F$ are not highly correlated and CS theory still holds empirically while exhibiting a sufficient degree of spatial correlation in the receptive field of a particular neuron as observed experimentally.

For an $\sqrt{n} \times \sqrt{n}$ pixel image, selecting the center of a receptive field is equivalent to randomly choosing coordinates on a $[1, \sqrt{n}] \times[1, \sqrt{n}]$ Cartesian grid, with each pair of integer coordinates corresponding to a different pixel location. The localized random sampling receptive field type specifies 


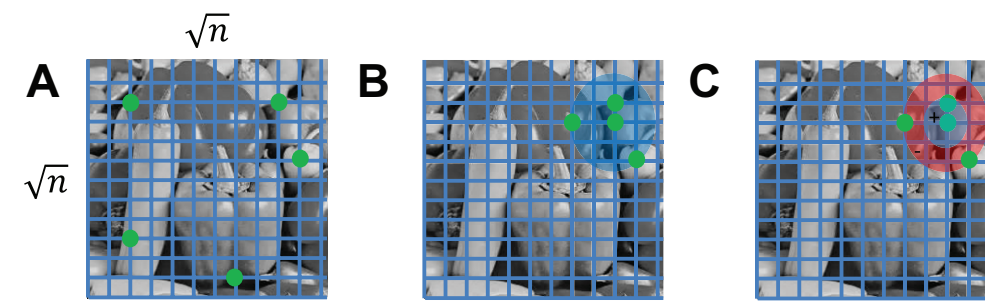

Figure 2: Schematic of receptive field models. Depicted are receptive fields sampling a $\sqrt{n} \times \sqrt{n}$ pixel image using (A) uniformly-random sampling, (B) localized random sampling, and (C) localized random center-surround sampling. For the given receptive field, sampled pixels are indicated by green circles. In (A) and (B), all samples are excitatory, whereas in $(\mathrm{C})$ samples in the inner circle are excitatory and all other samples are inhibitory.

that if the coordinates of the $i^{t h}$ receptive field center are $\left(x_{i}, y_{i}\right)$, then the probability, $P$, to sample a pixel with coordinates $\left(x_{j}, y_{j}\right)$ is given by

$$
P=\rho \exp \left(-\left[\left(x_{i}-x_{j}\right)^{2}+\left(y_{i}-y_{j}\right)^{2}\right] /\left[2 \sigma^{2}\right]\right),
$$

where $\rho$ represents the sampling probability if $\left(x_{i}, y_{i}\right)=\left(x_{j}, y_{j}\right)$, that is when the receptive field center matches the location of a given pixel, and $\sigma$ determines the distance over which the receptive field is expected to sample pixels. Each entry in a given row of $F$ is a Bernoulli random variable, determined independently of all other entries of $F$, with success probability given by Eq. 3 and connection strength $f$.

In the context of static image processing, previous work has demonstrated that, relative to uniformly random sampling, localized random sampling is robustly able to more accurately capture the dominant low and moderate frequency components composing an image [55]. This improvement garnered by localized random sampling was observed for a large ensemble of natural images over a broad parameter regime, particularly for moderate choices of receptive field size. In the next section, we will also investigate if the same improvements are garnered by localized random sampling in the case of reconstructing network inputs from evoked dynamics.

A final receptive field model, further physiologically refined, that we will consider is localized random center-surround sampling. We assume that the probability of sampling is still dictated by the localized random framework as in Eq. 3, but we now distinguish between excitatory and inhibitory connections. If a sample falls within a radius $r$ of the receptive field center, 
the connection is excitatory with strength $f_{E}>0$, whereas if the connection falls a distance greater than $r$ from the center, it is inhibitory with strength $f_{I}<0$. This framework captures the quintessential characteristics of an oncenter receptive field, which we consider for concreteness, while an off-center receptive field could be modeled analogously by reversing the excitatory and inhibitory regions.

\section{Results}

\subsection{Reconstruction of Natural Scenes}

To understand how evolution may have fine-tuned our sensory systems to process stimuli commonly encountered in the natural world, we first investigate how the various choices of receptive field model impact the reconstruction of natural scenes. In particular, using Eq. 2 we obtain a compressive sensing reconstruction, $p_{\text {recon }}$, of Fig. $1 \mathrm{C}$ using the downstream neuronal firing rates observed over a simulation time of $200 \mathrm{~ms}$, which is comparable to human reaction-times for visual stimuli $[61,62]$. For concreteness, we discuss a particular natural scene of size $100 \times 100$ pixels, though we obtain analogous results for alternative natural scenes of other sizes for comparable ratios of upstream to downstream neurons. In assessing how accurately a given image is encoded through the downstream network dynamics, we utilize the relative error $E=\left\|p-p_{\text {recon }}\right\|_{F} /\|p\|_{F}$ defined using the Frobenius matrix norm $\|p\|_{F}=\sqrt{\sum_{i} \sum_{j} p_{i j}^{2}}$. This gives a pixel-by-pixel comparison of the original image and reconstructed image. We seek the model parameter regimes which yield optimal encoding of natural stimuli and discuss their physiological implications.

For localized random sampling, we investigate the $(\rho, \sigma)$ parameter space, plotting the associated reconstruction error for each parameter choice in Fig. 3A. We observe that for moderately sized $\sigma$ and high $\rho$ the most accurate reconstructions are achieved with minimum relative error $E=0.208$ for $(\rho, \sigma)=(0.9,2.3)$. The optimal choice of $\sigma$, controlling the distance over which the receptive field spans, corresponds to moderately-sized receptive fields with an expected distance of approximately 3 pixels between a receptive field center and a sampled pixel. This agrees well with physiological receptive fields, which tend to sample spatially nearby pixel information over a relatively precise area of the visual field. The optimal choice of $\rho$, controlling the sampling density, leaves each pixel sampled approximately once 


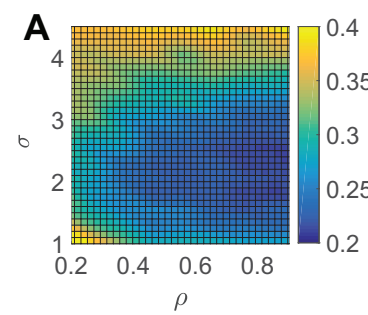

D

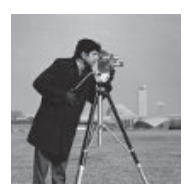

B

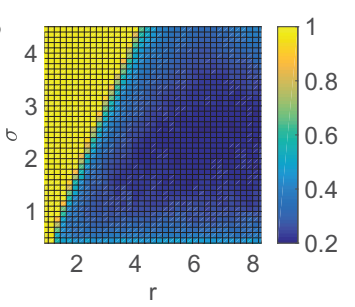

E
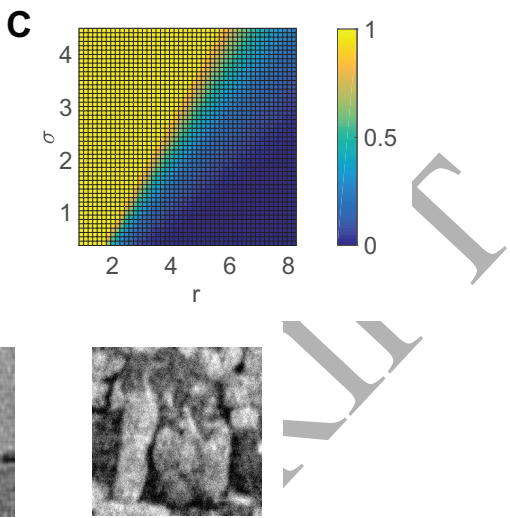

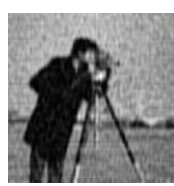

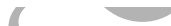

Figure 3: Parameterscape for CS reconstruction error in recovering the $100 \times 100$ pixel natural scene depicted in Fig. 1(C) using compressive sensing of the network dynamics. (A) Localized random sampling CS reconstruction error dependence on $(\rho, \sigma)$ parameter choice. The parameter choice yielding minimial error is $(\rho, \sigma)=(0.9,2.3)$ corresponding to relative error $E=0.208$. The excitatory feed-forward connection strength is $f=f_{E}=1$. (B) Localized random center-surround sampling CS reeonstruction error dependence on $(r, \sigma)$ parameter choice for $\rho=0.9$ and $f_{I}=0.25$. The parameter choice yielding minimial error is $(r, \sigma)=(8,2.0)$ corresponding to relative error $E=0.21$. (C) Ratio of inhibitory to excitatory connections in the feed-forward connectivity matrix corresponding to parameter choices in (B). (D) Sample natural scenes. (E) Localized random sampling reconstructions of the images in (D) using the optimal parameter choices and a $10: 1$ ratio of receptors to ganglion cells. Gray-scale images with pixel values given by integers in $[0,255]$ are utilized. We represent each entry of $p$ as the corresponding pixel value normalized by the sum of the sampled pixel values over the network such that the net input into a given downstream neuron is $\mathcal{O}(1)$.

with a connection density of $1 / m=0.999$ in the feed-forward connectivity matrix. This can be seen since the total number of entries in $F$ is $m n^{2}$ and thus $m n^{2}(1 / m)=n^{2}$ total pixels are measured. We note that for too large $\rho$ and $\sigma$ over-sampling results in each receptive field gathering information from too much of the visual field, such that there tends to be redundancy in the image information yielded by each receptive field. Likewise, for too small $\rho$ and $\sigma$, each receptive field does not gather enough spatially distinct information such that light intensity in certain locations may never be sampled and consequently less information will be available for reconstruction. For extreme values of $\rho$ near 0 or 1 , these problems are especially accentuated, yielding relative reconstruction errors near $E=1$, and thus we omit these parameter choices from Fig. 3A to focus on the error structure for more 
reasonable parameters.

For reference, we consider two sample natural scenes in Fig. 3D and exhibit their respective localized random sampling CS reconstructions in Fig. 3E for the optimal choice of $\rho$ and $\sigma$ for natural scene reconstruction. In each case, the rendered reconstruction captures the significant image features while losing only some small-scale structure typically near sharp edges embedded in low-amplitude high-frequency components potentially lost through the nonlinear network dynamics. We remark that for larger images with more pixels and generally more sparsity in the frequency domain, it is possible to reconstruct finer image details using the same ratio of downstream to upstream neurons. Similarly, using more ganglion cells relative to a fixed number of photoreceptors typically further increases reconstruction quality. In this study, we choose to utilize significantly fewer ganglion cells to align more closely with the significant disparity in the numbers of receptor cells and immediately downstream sensory neurons along the early stages of sensory pathways [46, 47]. Visual system models optimized for efficient coding suggest the spare sampling of stimuli by a relatively small pool of downstream sensory neurons may be the consequence of biological constraints on energy consumption and non-redundant information transmission [63].

It is important to note that certain parameter choices for localized random sampling are analogous to uniformly random sampling, giving a clear means to differentiate between how well the two receptive field models encode natural image information. For large $\sigma$ with moderate $\rho$, all pixels become equally likely to be sampled just as assumed in the uniformly-random sampling model. In Fig. 3A, we observe that for high $\sigma$ (i.e., $\sigma>3$ ), the CS reconstruction quality diminishes, indicating that spatially localized random sampling better encodes natural scene information. The reconstruction error dependence on sampling parameters using both uniformly random sampling and localized random sampling based on the recorded neuronal dynamics is analogous to the case of static CS reconstruction of natural scenes in the context of conventional image processing as studied in Ref. [55], demonstrating that the feed-forward network structure is primarily responsible for deviations in signal encoding quality and that image information is indeed well preserved through the network dynamics for natural scenes.

For localized random center-surround sampling, we similarly explore the $(r, \sigma)$ parameter space for natural scene inputs in Fig. 3B. We maintain the $\rho=0.9$ sampling density found in the optimal regime for localized random sampling, noting that as long as $\rho$ is sufficiently far from 0 or 1 , the CS re- 
construction quality is primarily determined by the other parameter choices. This can be seen in Fig. 3A for localized random sampling, and the same structure carries over to the case of center-surround sampling. For relatively large center radius $r \approx 8$ and moderate $\sigma \approx 2$, we observe the highest fidelity reconstructions using the center-surround localized random sampling. The extremum corresponds to a similarly large receptive field size as in the case of optimal localized random sampling with primarily excitatory feed-forward connections as indicated by the large optimal center radius.

To further analyze the role of the additional inhibitory connections garnered by the center-surround scheme, we plot in Fig. $3 C$ the ratio of the number of inhibitory to excitatory connections over the parameterscape. An increase in $r$ for fixed $\sigma$ results in a larger proportion of excitatory connections, which is to be expected since holding $\sigma$ constant fixes the size of the receptive field while increasing $r$ increases the percentage of these connections that are excitatory. It is important to remark that for $r$ sufficiently large, the center-surround structure reduces to purely excitatory connectivity, analogous to localized random sampling. In contrast, as $\sigma$ is increased with $r$ fixed, the proportion of inhibitory samples is increased, thereby keeping the total number of excitatory connections constant while increasing the number of inhibitory connections in F. Comparing Figs. 3B and 3C, we see that if the proportion of connections that are inhibitory is too large, the downstream neurons are over-inhibited, resulting in a lack of firing activity. Since the neuronal firing rates are used in Eq. 2 to reconstruct the input image, a degeneracy in firing neurons diminishes the reconstruction quality. We remark that we chose the inhibitory connection strength $f_{I}=0.25$, and by choosing alternative $f_{L}$ we simply shift the center radius yielding degenerate reconstructions $(E \approx 1)$. As $f_{I}$ is increased, for fixed receptive field size $\sigma$, the $r$ yielding a successful (non-degenerate) reconstruction will increase since more net excitation, stemming from increased center radius, is necessary to balance the increased inhibition so that the downstream neurons are able to fire. Once in the non-degenerate regime for a particular choice of $f_{I}$, we have verified empirically that analogous dependence on parameters is observed as in Fig. 3B.

In the presence of a relatively small proportion of inhibitory connections, we do observe natural scene reconstructions of comparable quality to purely excitatory localized random sampling for $\sigma$ moderate. However, any improvements garnered by the introduction of inhibitory connections are quite marginal relative to excitatory localized random sampling with the same $\sigma$. 
We hypothesize that a small number of inhibitory connections may increase the quality of edge reconstructions, but since edges are determined by low amplitude, high frequency components, the improvements in edge quality are largely masked by the quality of large-scale, low frequency information. We conclude that for natural scenes, both the localized random sampling and center-surround localized random sampling receptive field structures are comparable for encoding natural scene information in network dynamics, with the less physiological uniformly random sampling structure yielding relatively degraded representations of natural scene inputs. This result from our computational model analysis supports the notion that the physiological receptive field structure is indeed optimized through evolution for the encoding of natural stimuli through network dynamics.

\subsection{Reconstruction of Illusory Images}

While our previous analysis addresses images commonly encountered in the normal operating mode of a mammal, we now investigate how receptive field structure may impact the encoding of non-natural scenes, such as illusory images. Do the same optimal receptive field characteristics as in the case of natural scenes arise for illusory images or do these same characteristics contribute to the illusory effect since evolution has not optimized the processing of such stimuli?

For concreteness, we focus our analysis on the Hermann grid illusion in Fig. $1 \mathrm{~A}$ with $100 \times 100$ pixels, reconstructing this input image using compressive sensing of the downstream neuronal dynamics via localized random sampling and then center-surround localized random sampling. Using the same framework as in the previous section, we investigate the $(\rho, \sigma)$ parameter space for localized random sampling in Fig. 4A. We now observe a stark contrast to the case of natural scenes. For the grid illusion, a relatively small $\sigma \approx 0.8$ yields an optimal reconstruction with little dependence on the choice of $\rho$. The implication here is that a smaller receptive field size is necessary to achieve a high fidelity reconstruction of the Hermann grid. Likewise, in Fig. 4B, we analyze how the choice of $r$ and $\sigma$ impacts the reconstruction quality for the illusory image. As in the case of localized random sampling, we again observe that a relatively small choice of $\sigma \approx 0.8$ results in optimal encoding. We also observe that an optimal reconstruction is obtained when the center radius $r$ is relatively large, yielding a small proportion of inhibitory feed-forward connections or purely excitatory connections as in localized random sampling. The reasoning for the dependence on $r$ here is 

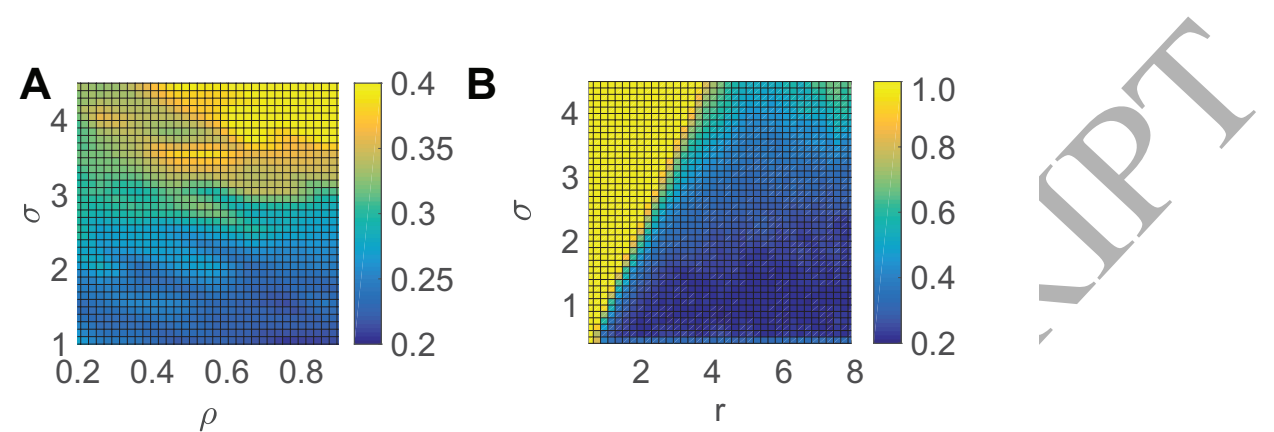

Figure 4: Parameterscape for CS reconstruction error in recovering the $100 \times 100$ pixel Hermann grid illusion depicted in Fig. 1(A) using compressive sensing of the network dynamics. (A) Localized random sampling CS reconstruction error dependence on $(\rho, \sigma)$ parameter choice. The parameter choice yielding minimial error is $(\rho, \sigma)=(0.9,0.8)$ corresponding to relative error $E=0.207$. (B) Localized random center-surround sampling CS reconstruction error dependence on $(r, \sigma)$ parameter choice. Unless specified otherwise, we assume $\rho=0.9, f_{E}=1$, and $f_{I}=0.25$ for the center-surround model. The parameter choice yielding minimial error is $(r, \sigma)=(4.2,0.8)$ corresponding to relative error $E=0.2$.

analogous to the case of natural scene inputs. Therefore, both the purely localized and center-surround random sampling models suggest that networks of neurons with small receptive fields, as observed for uniformly random sampling with low connection probability, most accurately encode the Hermann grid in network dynamics.

To further investigate the underlying cause for this optical illusion and why the dependence on sampling parameters for the $100 \times 100$ pixel Hermann grid is distinct from natural scenes, we exhibit several representative reconstructions for various choices of $r$ and $\sigma$ for localized random centersurround receptive fields in Fig. 5. In the degenerate case, when the proportion of inhibitory connections is too high (i.e., relatively low $r$ with high $\sigma)$, an/ indistinguishable reconstruction is obtained with large amounts of black space resulting from a deficiency in firing downstream neurons. In contrast, for low $\sigma \approx 0.5$, high fidelity reconstructions are achieved, with especially little error in the intersections, which are primarily white in the reconstructions. For intermediate receptive field size, $1.5 \lesssim \sigma \lesssim 2.5$ and sufficiently large $r$ such that the reconstruction is non-degenerate, we observe 


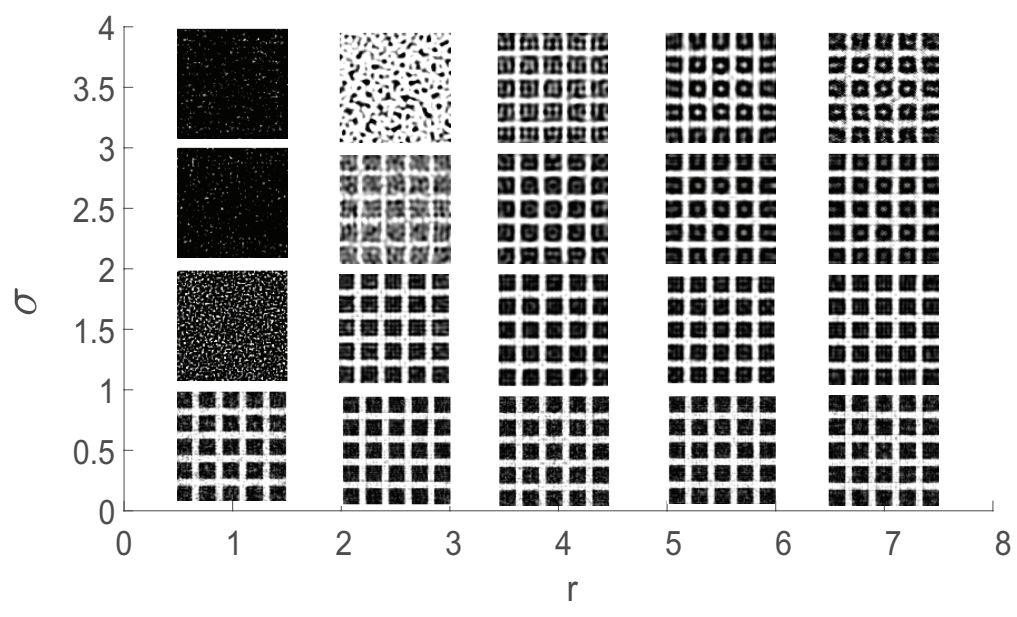

Figure 5: Sample reconstructions of the Hermann grid illusion using compressive sensing of the network dynamics over the localized random center-surround sampling parameter domain corresponding to $r=\{1,2.5,4,5.5,7\}$ and $\sigma=\{0.5,1.5,2.5,3.5\}$. For $r=7$, corresponding to primarily excitatory receptive fields over this parameter space, the mean reconstruction error in only the intersection reglons for the respective $\sigma$ values depicted is $0.137,0.138,0.148$, and 0.200 , respectively. For the same respective choices of $\sigma$, the difference between the mean pixel value within the reconstructed intersections and the mean pixel value along the reconstructed lines is 12.1,16.2,17.5, and 22.0, respectively. All errors were measured using the Frobenius norm, as specified in Sec. 3.1.

degraded, though decipherable, reconstructions with increased error in the intersection regions, marked by darker pixels. This suggests that the illusion is manifesting from the spatial localization in the receptive field. In fact, darker intersection regions as well as degraded overall reconstruction quality is observed regardless of whether or not an inhibitory surround region is included, underlining the spatial localization as opposed to center-surround structure as a primary cause. As $\sigma$ is increased further to $\sigma \approx 3.5$ the fundamental impact of receptive field size becomes more pronounced, with the receptive field becoming so large that even in the non-intersection regions white pixels are introduced from over-sampling nearby white intersections. To demonstrate a similar dependence on receptive field size for purely localized random sampling in the absence of inhibitory connections, we plot in Fig. 6A-D sample reconstructions as $\sigma$ is increased. We again observe the darkening of intersection pixels akin to the illusory effect for moderate $\sigma$ with degraded reconstruction quality throughout the image for large re- 


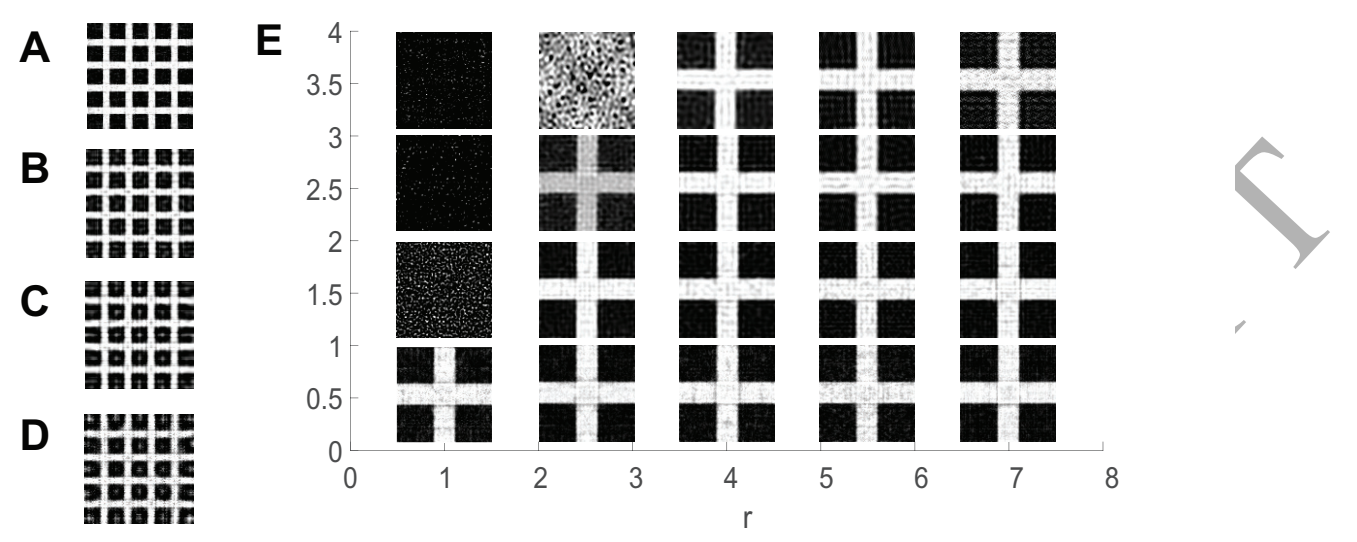

Figure 6: Encoding dependence on receptive field size. (A)-(D) Sample reconstructions of the Hermann grid illusion in Fig. 1(A) using localized random sampling for $\rho=0.9$ and $\sigma=0.5,1.5,2.5$, and 3.5, for (A)-(D), respectively. The corresponding reconstruction errors for the full Hermann grid are 0.216, 0.243, 0.27, and 0.387, respectively. (E) Sample reconstructions of a single $100 \times 100$ pixel intersection of horizontal and vertical white lines on a black background over the localized random center-surround sampling parameter domain corresponding to $r=\{1,2.5,4,5.5,7\}$ and $\sigma=\{0.5,1.5,2.5,3.5\}$. This sensory input is analogous to viewing a single intersection of the Hermann grid illusion in the visual field. For $r=7$, corresponding to primarily excitatory receptive fields over this parameter space, the mean reconstruction error in only the intersection regions for the respective $\sigma$ values depicted is $0.100,0.090,0.094$, and 0.140 , respectively. For the same respective choices of $\sigma$, the difference between the mean pixel value within the reconstructed intersections and the mean pixel value along the reconstructed lines is 9.5,9.6,9.1, and 9.2, respectively. All errors were measured using the Frobenius norm, as specified in Sec. 3.1.

ceptive field size; only for sufficiently small receptive field size is the illusory effect minimized. Intuitively, as the receptive field size is increased beyond the size of the intersection region, increased reconstruction error is incurred. This agrees with the parameterscape plots depicted by Fig. 4 in that for both purely excitatory and center-surround localized random sampling, the reconstruction quality demonstrates a comparable dependence on receptive field size, with larger receptive fields diminishing reconstruction quality regardless of the presence of additional surround inhibition.

To further illustrate the importance of the relative size of the intersection compared to the receptive field size in producing the illusory effect, we turn to considering the case in which only a single $100 \times 100$ intersection is in the field of view. We study representative reconstructions of the intersection image 
as $r$ and $\sigma$ are varied in the localized random center-surround receptive field model in Fig. 6E. Just as observed for natural images and the full Hermann grid, degenerate reconstructions are obtained for sufficiently large $\sigma$ and small $r$. However, in the non-degenerate case, we see that even for large $\sigma \approx 3.5$, the pixels in the intersection primarily match the pixels elsewhere along the vertical and horizontal lines. Comparing the reconstruction error across only the intersection regions, as listed in the captions of Figs. 5 and 6, we observe a marked decrease in error when viewing only a single intersection. The mean pixel value within the reconstructed intersections and the mean pixel value along the reconstructed lines are nearly identical across the receptive field sizes investigated when viewing a single intersection, whereas this discrepancy increases with receptive field size when viewing the full Hermann grid. When the receptive field size is still small relative to the size of the intersection in the visual field, little illusory effect is observed. The Hermann grid illusion is known to be diminished in the center of view [27, 28], and likely this is due to the particularly small receptive fields of gangtion cells in the fovea. Only when the receptive field size becomes comparable to the size of an intersection does the illusory effect begin to arise.

We underline two primary sources of error in the neuronal encoding of sensory inputs, namely the reeeptive field structure and the input-output relationship between the neuronal firing rates and visual stimulus underlying the nonlinear network dynamics given by Eq. 2. With the goal of isolating the role of network dynamics in optical illusions, we study the reconstruction of the Hermann grid illusion using static compressive sensing. To consider the related image processing problem, we reconstruct the grid image using a sampling matrix with the same number of rows and same structure as the feed-forward connectivity matrix $F$ in our neuronal network model. This produces an underdetermined linear system relating the sampled image data $b$ and the unknown sampled image $p$ of form $F p=b$ analogous to Eq. 2. A key difference is that in the static case no error is produced by the linear system itself and all error manifests from sampling, whereas in the case of network dynamics, there is inherently error in using the approximate inputoutput mapping which may potentially compound the error produced as a result of the receptive field structure.

Varying center radius $r$ and receptive field size $\sigma$ in the localized random center-surround receptive field model determining $F$, we plot representative reconstructions in Fig. 7A using static CS. Note that since our earlier discussion highlighted localization as the key structural cause of the illusion, we 

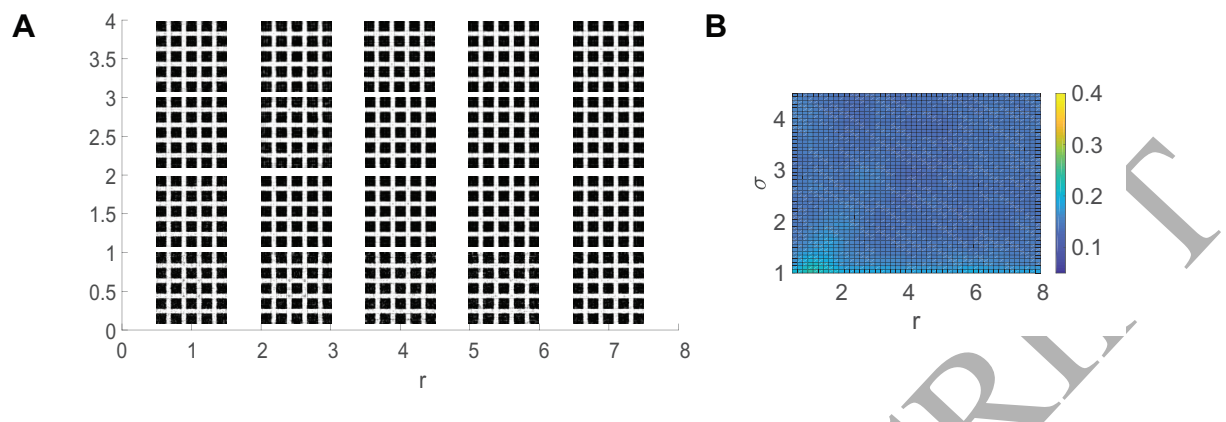

Figure 7: Static compressive sensing reconstructions of the Hermann grid. A. Sample reconstructions of the Hermann grid illusion using static compressive sensing over the localized random center-surround sampling parameter domain corresponding to $r=$ $\{1,2.5,4,5.5,7\}$ and $\sigma=\{0.5,1.5,2.5,3.5\}$. For $r=7$, corresponding to primarily excitatory receptive fields over this parameter space, the mean reconstruction error in only the intersection regions for the respective $\sigma$ values depicted is $0.119,0.134,0.134$, and 0.114 , respectively. B. Static localized random center-surround sampling CS reconstruction error dependence on $(r, \sigma)$ sampling parameter space. The minimal reconstruction error for the full Hermann grid is $E=0.13$. All errors were measured using the Frobenius norm, as specified in Sec. 3.1 .

focus our discussion here on comparing the success of reconstructions using static CS and CS based on network dynamics so as to isolate the impact of network dynamics. Several significant differences from the framework using compressive sensing of network dynamics arise. First, there is no longer a degenerate regime since neurons becoming over inhibited and failing to fire is no longer an issue in the case of static CS. The illusory effect is also reduced in the absence of network dynamics, with the pixels in both the intersection and non-intersection locations relatively well matched. In Fig. 7B, we depict the reconstruction error using static localized random center-surround sampling over the $(r, \sigma)$ parameter space. We see improved reconstruction quality relative to even the optimal analogous network dynamics CS reconstructions, yielding an approximately halved minimal reconstruction error for the full Hermann grid. With the reconstruction error in the static case demonstrating little dependence on the choice of sampling parameters, a combination of the nonlinear dynamics and localization potentially together contribute to the errors observed in the case of the neuronal network model.

Since the Hermann grid image is largely periodic in its spatial structure with large local regions of approximately identical pixels, a high quality static 
A

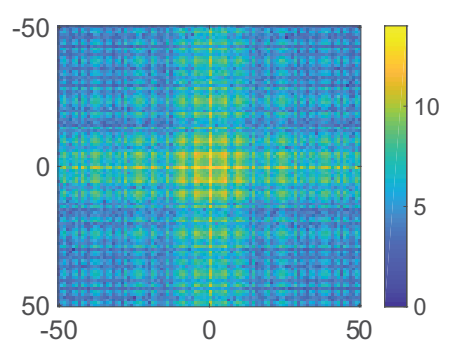

B

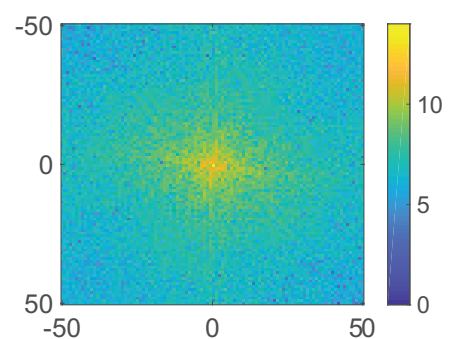

Figure 8: Sparse representation of stimuli. Two-dimensional discrete-cosine transform of (A) the Hermann grid image in Fig. 1A and (B) the peppers image in Fíg. 1C. Each representation is computed from the natural logarithm of the absolute value of the twodimensional discrete-cosine transform of each image, emphasizing the detailed structure of lower amplitude frequency components.

CS reconstruction is achievable over a broad parameter regime encompassing all three receptive field structures discussed in this work. In the frequency domain, the dominant low-frequency components of the Hermann grid image are primarily harmonics of the fundamental frequency of the vertical and horizontal lines, as demonstrated by its frequency domain representation in Fig. 8A, which implies that a high fidelity reconstruction is primarily achievable by capturing the fundamental frequencies. This contrasts from the frequency domain representation of a natural scene, as given for the peppers image in Fig. 8B, which, although is largely composed of dominant low frequency modes, primarily demonstrates aperiodicity in its frequency amplitude structure. These significant structural differences between natural and non-natural scenes lead to distinct optimal receptive field structures.

We expect that for relatively large receptive field sizes, the underlying nonlinear neuronal dynamics evoked from an input stimulus may yield insufficient information regarding image structure particularly when locally sampled pixels yield a firing rate that misaligns with the light intensity in its receptive field center. For example, even if the receptive field of a neuron samples several white pixels it may still sample such a relatively large number of black pixels that it will never fire in response to a stimulus, thereby yielding no information regarding the local level of whiteness. A cohort of such uninformative single neuron representations with nearby centers could thus completely misrepresent the local image structure, such as in the illusory region of the Hermann grid image. In contrast, since natural stimuli gen- 
erally have smoother edges, the relatively large spatial correlation between neighboring pixels diminishes the likelihood of this degeneracy for moderately sized receptive fields.

Considering a high fidelity reconstruction is generally achieved regardless of receptive field size using static CS, we conclude that, in the context of our model network, error induced by the nonlinear dynamics and related approximate input-output mapping are crucial to causing the illusion. A slightly distorted representation of image information stemming from the imperfect linear map used in the network dynamics reconstruction causes the sampled data to loose the particularly simple structure that made the static CS reconstruction succeed especially well. This is further evidenced by the robust reconstructions of the Hermann grid achieved over the entire sampling parameter space using static CS as shown in Fig. 7B.

\section{Discussion}

Using an idealized computational model of the early visual system, we investigated the impact of receptive field structure on the encoding of stimuli through neuronal dynamics. We demonstrated that for natural scenes, receptive fields sampling pixels over a small but spatially localized region of the visual field yield particularly high fidelity image representations, even in the presence of lateral inhibition as found in the physiological center-surround structure. For the Hermann grid illusion, on the other hand, the role of spatial localization was largely reversed. For even moderately-sized receptive fields, the image reconstruction from neuronal dynamics was degraded, with pronounced darkening of the intersections where the grid illusion is known to occur. The illusory effect materialized in the reconstruction for both purely excitatory/and center-surround localized random sampling, underlining the role of spatial localization in sampling in producing the illusion. For particularly small receptive fields, such as those of ganglion cells in the fovea, the most accurate reconstructions of the Hermann grid were achieved, agreeing with human observation that the illusory effect is diminished in the center of view. Through direct comparison to analogous reconstructions using static compressive sensing and a perfect linear encoder, we further demonstrated that image information lost though nonlinear neuronal dynamics and an imperfect embedded linear input-output mapping fundamentally contributes to the manifestation of the illusion. The classical hypothesis that Hermann grid-type illusions primarily arise due to center-surround sampling is intuitive 
though largely circumvents the specific impact of neuronal dynamics and may be over-restrictive in requiring surround sampling in order to produce the illusory effect. Our work shows that only a combination of spatial localization in receptive fields combined with encoding error induced by the nonlinear neuronal dynamics is potentially sufficient to account for such illusions.

This novel connection between model neuronal network structure, nonlinear dynamics, and input encoding highlights several new directions for future study. While we have focused our analysis on the Hermann grid illusion in particular, the compressive-sensing framework developed could be utilized to study the root causes of other classes of optical illusions. For example, the Mach bands illusion has long been hypothesized to be a result of the center-surround receptive field structure [25, 64], however a more systematic computational investigation would likely yield additional insights and potentially demonstrate an encoding structure similar to the Hermann grid illusion investigated in detail here. Though we argue in the context of the derived input-output mapping given by Eq. 2 that read-out error based on the nonlinear network dynamics fundamentally eontributes to Hermann gridtype illusions, it would also be informative to determine if other embedded static input-output transformations yield analogous effects and also if similar trends hold for alternative network modéls.

We specifically utilize an idealized network model to focus on potential mechanisms underlying degeneracies in the processing of non-natural scenes. While the early visual system may demonstrate a host of additional properties, such as diversity in receptive field structure, more detailed spatial receptive field architecture, direction or orientation selectivity, and timedelayed responses to stimuli, more complex models may obscure underlying mechanistic insights. Hence, we focus on several key properties that together potentially explain specific illusory effects. In this work we had assumed the receptive field size to be homogeneous in our model network, though experimental eyidence indicates that receptive fields demonstrate diversity in size and depend on neuron type [18-20]. It remains to be fully understood how receptive field size diversity impacts the encoding of sensory stimuli and compressive sensing reconstruction in general, and model investigation could determine if there is potentially an optimal distribution of receptive field sizes. Our analysis demonstrates that center-surround sampling well preserves dominant frequency mode information for natural scenes, but a more thorough analysis of how the center-surround structure may improve the representation of edge information, encoded by more subtle higher fre- 
quency components, is still necessary. Furthermore, considering vertebrates often possess parallel visual channels [65] and thus incomplete image information may be encoded in any given channel, utilizing block or distributed compressive sensing in a manner analogous to our developed framework may yield new insights into the functional roles of parallel processing in sensory systems $[66,67]$. We expect that downstream visual processing via additional receptive field types would further improve natural scene encoding, particularly with respect to higher order stimulus features. Reflecting the architecture of the primary visual cortex by including an ensemble of simple cells in our modeling framework [68, 69], via Gabor functions for example $[16,70-72]$, would produce orientation selectivity in sampling. The resultant diversity in receptive field elongation and orientation would likely facilitate the CS reconstruction of a broader class of oriented edges and corresponding high frequency modes composing natural scenes.

It was recently hypothesized, using various perturbations of the original Hermann grid illusion, that the conventional explanation of the illusory effect may be incomplete [73]. One such adjustment is that by slanting the white lines while preserving the white-space gain in the area around the intersections, a decrease in the illusory effect is produced. This suggests that the illusion may also depend on orientation-selectivity beyond the spatially localized structure. While not verified rigorously, orientation-specific neurons in downstream layers of the visual system [74] may explain these deviations in the illusory effect, and the root cause is an interesting area for future investigation.

\section{Acknowledgements}

This work was supported by NSF grant DMS-1812478 and a Swarthmore Faculty Research Support Grant.

\section{References}

[1] D. J. Field, What is the goal of sensory coding?, Neural Computation 6 (4) (1994) 559-601. doi:10.1162/neco.1994.6.4.559.

[2] J. H. Kaas, The evolution of complex sensory systems in mammals, J. Exp. Biol. 146 (1989) 165-176. 
[3] J. H. Kaas, The evolution of the complex sensory and motor systems of the human brain, Brain Res. Bull. 75 (2-4) (2008) 384-390.

[4] H. B. Barlow, The coding of sensory messages, in: W. H. Thorpe, O. L. Zangwill (Eds.), Current Problems in Animal Behaviour, Cambridge University Press, 1961, pp. 331-360.

[5] H. Barlow, Redundancy reduction revisited., Network 12 (3) (2001) 241253.

[6] T. N. Wiesel, Receptive fields of ganglion cells in the cat's retina, J Physiol 153 (1960) 583-594.

[7] D. H. Hubel, T. N. Wiesel, Receptive fields of optic nerve fibres in the spider monkey., J Physiol 154 (1960) 572-580.

[8] M. S. Graziano, C. G. Gross, A bimodal map of space: somatosensory receptive fields in the macaque putamen with corresponding visual receptive fields, Exp Brain Res 97 (1) (1993) 96-109.

[9] D. A. Wilson, Receptive fields in the rat piriform cortex, Chem. Senses 26 (5) (2001) 577-584.

[10] C. Welker, Receptive fields of barrels in the somatosensory neocortex of the rat, J. Comp. Neurol. 166 (2) (1976) 173-189.

[11] K. Mori, H. Nagao, Y. Yoshihara, The olfactory bulb: coding and processing of odor molecule information, Science 286 (5440) (1999) 711-715.

[12] E. I. Knudsen, M. Konishi, Center-surround organization of auditory receptive fields in the owl, Science 202 (4369) (1978) 778-780.

[13] D. L. Ringach, M. J. Hawken, R. Shapley, Receptive field structure of neurons in monkey primary visual cortex revealed by stimulation with natural image sequences, J Vis 2 (1) (2002) 12-24.

[14] R. L. De Valois, D. G. Albrecht, L. G. Thorell, Spatial frequency selectivity of cells in macaque visual cortex, Vision Res. 22 (5) (1982) $545-559$.

[15] J. B. Levitt, D. C. Kiper, J. A. Movshon, Receptive fields and functional architecture of macaque V2, J. Neurophysiol. 71 (6) (1994) 2517-2542. 
[16] J. H. Elder, A. J. Sachs, Psychophysical receptive fields of edge detection mechanisms, Vision Res. 44 (8) (2004) 795-813.

[17] C. Enroth-Cugell, J. G. Robson, The contrast sensitivity of retinal ganglion cells of the cat, J. Physiol. (Lond.) 187 (3) (1966) 517-552.

[18] D. Hubel, Eye, Brain, and Vision, Scientific American Library Series, Henry Holt and Company, New York, 1995.

[19] M. P. Sceniak, D. L. Ringach, M. J. Hawken, R. Shapley, Contrast's effect on spatial summation by macaque V1 neurons, Nat. Neurosci. 2 (8) (1999) 733-739.

[20] R. Desimone, T. D. Albright, C. G. Gross, C. Bruce, Stimulus-selective properties of inferior temporal neurons in the macaque, J. Neurosci. 4 (8) (1984) 2051-2062.

[21] B. M. Dow, A. Z. Snyder, R. G. Vautin, R. Bauer, Magnification factor and receptive field size in foveal striate cortex of the monkey, Exp Brain Res 44 (2) (1981) 213-228.

[22] N. Drasdo, C. L. Millican, C. R. Katholi, C. A. Curcio, The length of henle fibers in the human retina and a model of ganglion receptive field density in the visual field, Vision research 47 (22) (2007) 2901-2911.

[23] V. Balasubramanian, P. Sterling, Receptive fields and functional architecture in the retina, J. Physiol. (Lond.) 587 (Pt 12) (2009) 2753-2767.

[24] M. A. Silver, S. Kástner, Topographic maps in human frontal and parietal cortex, Trends Cogn. Sci. (Regul. Ed.) 13 (11) (2009) 488-495.

[25] D. M. Eagleman, Visual illusions and neurobiology, Nat. Rev. Neurosci. 2 (12) , (2001) 920-926.

[26] L. Hermann, Eine erscheinung simultanen contrastes, Pflügers Archiv European Journal of Physiology 3 (1) (1870) 13-15.

[27] G. Baumgartner, Indirekte größenbestimmung der rezeptiven felder der retina beim menschen mittels der hermannschen gittertäuschung, Pflügers Archiv European Journal of Physiology 272 (1) (1960) 21-22. 
[28] L. Spillmann, The Hermann grid illusion: a tool for studying human perspective field organization, Perception 23 (6) (1994) 691-708.

[29] V. J. Barranca, G. Kovačič, D. Zhou, D. Cai, Sparsity and compressed coding in sensory systems, PLoS Comput. Biol. 10 (8) (2014) e1003793.

[30] S. M. Wu, Synaptic organization of the vertebrate retina: general principles and species-specific variations: the friedenwald lecture., Invest Ophthalmol Vis Sci 51 (3) (2010) 1263-1274. doi:10.1167/iovs.09-4396.

[31] A. Corral, C. J. Pérez, A. Díaz-Guilera, A. Arenas, Self-orgamized criticality and synchronization in a lattice model of integrate-and-fire oscillators., Phys. Rev. Lett. 74 (1) (1995) 118-121.

[32] W. Mather, M. R. Bennett, J. Hasty, L. S. Tsimring, Delay-induced degrade-and-fire oscillations in small genetic circuits, Phys. Rev. Lett. 102 (6) (2009) 068105.

[33] V. J. Barranca, D. C. Johnson, J. L. Moyher, J. P. Sauppe, M. S. Shkarayev, G. Kovačič, D. Cai, Dynamics of the exponential integrateand-fire model with slow currents and adaptation, J. Comput. Neurosci. 37 (1) (2014) 161-180.

[34] A. V. Rangan, D. Cai, Maximum-entropy closures for kinetic theories of neuronal network dynamics, Phys. Rev. Lett. 96 (17) (2006) 178101.

[35] D. A. McCormick, B. W. Connors, J. W. Lighthall, D. A. Prince, Comparative electrophysiology of pyramidal and sparsely spiny stellate neurons of the neocortex, J. Neurophysiol. 54 (4) (1985) 782-806.

[36] M. Shelley, D. McLaughlin, R. Shapley, J. Wielaard, States of high conductance in a large-scale model of the visual cortex, J. Comp. Neurosci. 13 (2002) 93-109.

[37] G. Kovačič, L. Tao, A. V. Rangan, D. Cai, Fokker-planck description of conductance-based integrate-and-fire neuronal networks, Phys. Rev. E 80 (2009) 021904.

[38] R. Brette, M. Rudolph, T. Carnevale, M. Hines, D. Beeman, J. M. Bower, M. Diesmann, A. Morrison, P. H. Goodman, F. C. Harris Jr, M. Zirpe, T. Natschlager, D. Pecevski, B. Ermentrout, M. Djurfeldt, 
A. Lansner, O. Rochel, T. Vieville, E. Muller, A. P. Davison, S. E. Boustani, A. Destexhe, Simulation of networks of spiking neurons: A review of tools and strategies, J. Comput. Neurosci. 23 (3) (2007) 349 398.

[39] W. Dai, M. A. Sheikh, O. Milenkovic, R. G. Baraniuk, Compressive sensing DNA microarrays, J. Bioinform. Syst. Biol. (2009) 162824.

[40] S. Cocco, S. Leibler, R. Monasson, Neuronal couplings between rétinal ganglion cells inferred by efficient inverse statistical physics methods, Proc. Natl. Acad. Sci. U.S.A. 106 (33) (2009) 14058-14062.

[41] D. J. DeBoer, D. I. Vaney, Gap-junction communication between subtypes of direction-selective ganglion cells in the developing retina, J. Comp. Neurol. 482 (1) (2005) 85-93.

[42] P. K. Trong, F. Rieke, Origin of correlated activity between parasol retinal ganglion cells, Nat. Neurosci. 11 (11) (2008) 1343-1351.

[43] S. Trenholm, D. J. Schwab, V. Balasubramanian, G. B. Awatramani, Lag normalization in an electrically coupled neural network, Nat. Neurosci. 16 (2) (2013) 154-156. doi:10.1038/nn.3308.

[44] S. A. Bloomfield, B. Nolgyi, The diverse functional roles and regulation of neuronal gap junetions in the retina, Nat. Rev. Neurosci. 10 (7) (2009) 495-506.

[45] F. A. Dunn, F. Rieke, The impact of photoreceptor noise on retinal gain controls, Curr. Opin. Neurobiol. 16 (4) (2006) 363-370.

[46] H. B. Barlow, The ferrier lecture, 1980. critical limiting factors in the design of the eye and visual cortex., Proc R Soc Lond B Biol Sci 212 (1186) (1981) $1-34$.

[47] L.) B. Buck, Information coding in the vertebrate olfactory system., Annu Rev Neurosci 19 (1996) 517-544. doi:10.1146/annurev.ne.19.030196.002505.

[48] A. Treves, Mean field analysis of neuronal spike dynamics, Network 4 (1993) 259-284. 
[49] R. Ben-Yishai, R. Bar-Or, H. Sompolinsky, Theory of orientation tuning in the visual cortex, Proc Nat Acad Sci USA 92 (1995) 3844-3848.

[50] V. J. Barranca, D. Zhou, D. Cai, Compressive sensing reconstruction of feed-forward connectivity in pulse-coupled nonlinear networks, Phys Rev E 93 (6) (2016) 060201.

[51] C. E. Shannon, Communication in the Presence of Noise, Proceedings of the IRE 37 (1) (1949) 10-21.

[52] E. J. Candes, J. K. Romberg, T. Tao, Stable signal recovery from incomplete and inaccurate measurements, Communications on Pure and Applied Mathematics 59 (8) (2006) 1207-1223.

[53] E. J. Candes, M. B. Wakin, An Introduction To Compressive Sampling, Signal Processing Magazine, IEEE 25 (2) (2008) 21-30.

[54] J. A. Tropp, A. C. Gilbert, Signal Recovery From Random Measurements Via Orthogonal Matching Pursuit, IEEE Transactions on Information Theory 53 (12) (2007) 4655-4666.

[55] V. J. Barranca, G. Kovačič, D. Zhou, D. Cai, Improved Compressive Sensing of Natural Scenes Using Localized Random Sampling, Sci Rep 6 (2016) 31976.

[56] A. Haar, Zur Theorie der orthogonalen Funktionensysteme, Mathematische Annalen 69 (3) (1910) 331-371.

[57] C. E. Heil, D. F. Walnut, Continuous and discrete wavelet transforms, SIAM review 31 (4) (1989) 628-666.

[58] D. L. Donoho, Y. Tsaig, Fast solution of-norm minimization problems when the solution may be sparse, IEEE Transactions on Information Theory 54 (11) (2008) 4789-4812.

[59] R. Baraniuk, Compressive sensing, IEEE Signal Processing Mag (2007) 118-120.

[60] W. S. Geisler, Visual perception and the statistical properties of natural scenes, Annu Rev Psychol 59 (2008) 167-192. 
[61] K. Amano, N. Goda, S. Nishida, Y. Ejima, T. Takeda, Y. Ohtani, Estimation of the timing of human visual perception from magnetoencephalography, J. Neurosci. 26 (15) (2006) 3981-3991.

[62] S. Ando, Y. Yamada, M. Kokubu, Reaction time to peripheral visual stimuli during exercise under hypoxia, J. Appl. Physiol. 108 (5)(2010) $1210-1216$.

[63] E. Doi, J. L. Gauthier, G. D. Field, J. Shlens, A. Sher, M. Greschner, T. A. Machado, L. H. Jepson, K. Mathieson, D. E. Gunning, A. M. Litke, L. Paninski, E. J. Chichilnisky, E. P. Simoncelli, Efficient coding of spatial information in the primate retina, J. Neurosci. 32 (46) (2012) 16256-16264.

[64] F. Ratliff, H. K. Hartline, The responses of Limulus optic nerve fibers to patterns of illumination on the receptor mosaic, J. Gen. Physiol. 42 (6) (1959) 1241-1255.

[65] T. Baden, P. Berens, K. Franke, M. Roman Roson, M. Bethge, T. Euler, The functional diversity of retinal ganglion cells in the mouse, Nature 529 (7586) (2016) 345-350,

[66] L. Gan, Block compressed sensing of natural images, in: 2007 15th International Conference on Digital Signal Processing, 2007, pp. 403406. doi:10.1109/1CDSP.2007.4288604.

[67] M. F. Duarte, S. Sarvotham, D. Baron, M. B. Wakin, R. G. Baraniuk, Distributed compressed sensing of jointly sparse signals, in: Conference Record of the Thirty-Ninth Asilomar Conference onSignals, Systems and Computers, 2005., 2005, pp. 1537-1541. doi:10.1109/ACSSC.2005.1600024.

[68] D. H. Hubel, T. N. Wiesel, Receptive fields of single neurones in the cat's striate cortex., J Physiol 148 (1959) 574-591.

[69] C. M. Niell, Cell types, circuits, and receptive fields in the mouse visual cortex, Annu. Rev. Neurosci. 38 (2015) 413-431.

[70] J. G. Daugman, Two-dimensional spectral analysis of cortical receptive field profiles, Vision Res. 20 (10) (1980) 847-856. 
[71] S. Marcelja, Mathematical description of the responses of simple cortical cells, J Opt Soc Am 70 (11) (1980) 1297-1300.

[72] J. P. Jones, L. A. Palmer, An evaluation of the two-dimensional Gabor filter model of simple receptive fields in cat striate cortex, J. Neurophysiol. 58 (6) (1987) 1233-1258.

[73] P. H. Schiller, C. E. Carvey, The Hermann grid illusion revisited, Perception 34 (11) (2005) 1375-1397.

[74] T. Bonhoeffer, A. Grinvald, Iso-orientation domains in cat visual cortex are arranged in pinwheel like patterns, Nature 353(1991) 429-431. 